\title{
Development of a ReaxFF description for gold
}

\author{
T. T. Järvi ${ }^{1}$, A. Kuronen ${ }^{1}$, M. Hakala ${ }^{1}$, K. Nordlund ${ }^{1}$, A. C. T. van Duin ${ }^{2}$, W. A. Goddard III ${ }^{2}$, and T. Jacob ${ }^{3}$ \\ ${ }^{1}$ Department of Physics, University of Helsinki, P.O. Box 43, FI-00014 Helsinki, Finland \\ 2 Materials and Process Simulation Center, California Institute of Technology, Pasadena CA 91125, USA \\ ${ }^{3}$ Institut für Elektrochemie, Universität Ulm, Albert-Einstein-Allee 47, D-89081 Ulm, Germany
}

September 23, 2008

\begin{abstract}
Atomistic simulations of the chemistry of thiol-gold-systems have been restricted by the lack of interatomic interaction models for the involved elements. The ReaxFF framework already has potentials for hydrocarbons, making it an attractive basis for extending to the complete AuSCH-system. Here, an interatomic potential for gold, based on the ReaxFF framework, is presented and compared to existing gold potentials available in the literature.
\end{abstract}

PACS. 34.20.Cf Interatomic potentials and forces - 02.70.Ns Molecular dynamics and particle methods -68.35.-p Solid surfaces and solid-solid interfaces: structure and energetics

\section{Introduction}

Self-assembled monolayers of thiols, usually on gold surfaces, are widely explored systems due to their applications in many areas of nanotechnology (see ref. [1] and references therein). The gold-sulphur bond provides a link between the metal and the molecules, allowing the monolayers to be used for, e.g., electron transfer through organic systems or as biological sensors. However, atomistic simulations of gold-thiol systems have so far been restricted by the lack of interatomic potentials. A few potential energy surface expressions for different thiols on gold have been constructed [2,3], and Au-S pair potentials have been used with non-reactive force fields $[4,5]$. The only fully reactive atomistic simulation method available has been density functional theory. It is, however, prohibitively slow for dynamical studies of systems of several hundreds of atoms, especially on extended time scales. Development of classical potentials is therefore required.

An attractive basis for developing a full set of potentials for the gold-thiol system is the ReaxFF framework that already has potentials for hydrocarbons [6,7] and has 
also been used for metals $[8,9,10,11]$. It also has the advantage of being able to treat Coulomb interactions.

To this end, we presently introduce a ReaxFF potential for gold and give a review of other available gold potentials.

\section{Force field optimization}

The ReaxFF potential functions and fitting methods have been described in detail elsewhere (see refs. [6,12]). For a single metallic element, it has been shown $[10,9]$ that one already obtains a good description with a subset of possible interactions, the total energy of the system consisting of three terms,

$$
E_{\text {system }}=E_{\text {bond }}+E_{\text {over }}+E_{\mathrm{vdW}}
$$

with $E_{\text {bond }}$ and $E_{\mathrm{vdW}}$ describing bonds and van der Waals interactions, respectively, and $E_{\text {over }}$ correcting for possible over-coordinations.

Here we present a potential for gold, optimized against a database of $a b$ initio density functional theory (DFT) and experimental values for energies and bond lengths of various bulk and surface systems and the dimer, discussed in detail later. The potential functions and parameters are detailed in appendix A. A potential input file is also available as supporting information for this article.

Most of the $a b$ initio data were produced using the Vienna ab initio simulation package (VASP) $[13,14,15,16]$ and the plane-augmented-wave method and pseudopotentials $[17,18]$ in the local density approximation (LDA). LDA was found to give a better description of the lat- tice constant and bulk modulus of gold than what was obtained with the PBE gradient correction [19]. However, since PBE describes small clusters better, as demonstrated in refs. $[20,21,22]$, it was used for the dimer. These calculations were done using Gaussian 03 [23] with a basis set described in ref. [24] (basis III). The LDA and PBE results of several systems are compared in detail below, where the potential properties are discussed.

In the following section we detail the new potential's properties while simultaneously reviewing existing Au potentials available in literature. The existing potentials considered are summarized in table 1 . Of the corrected effective medium method (CEM) potential we used a version that was smoothly joined to the universal repulsive Ziegler-Biersack-Littmark potential [25] at short distances. This modification does not change the equilibrium properties, though.

\begin{tabular}{ll} 
potential & ref. \\
\hline Foiles et al. (FBD) & {$[26]$} \\
Glue model & {$[27]$} \\
MD/MC-CEM (CEM) & {$[28,29,30]$} \\
Voter & {$[31]$} \\
Cleri-Rosato (C-R) & {$[32]$} \\
Cai and Ye (CY) & {$[33]$} \\
Kallinteris et al. (KAL) & {$[34]$} \\
Chamati and Papanicolaou (CP) & {$[35]$} \\
Grochola et al. (GRS) & {$[36]$}
\end{tabular}

Table 1. Existing potentials for gold that were used for comparison. 


\section{Results and discussion}

\subsection{Bulk phases}

For the bulk phases of gold (fcc, hcp, bcc, sc, and diamond), the $a b$ initio equations of state were included in the fitting database. Figures 1 and 2 show the energyvolume curves for the phases, while table 2 summarizes the main bulk properties as evaluated with the optimized potential and a comparison to corresponding experimental and DFT values.

The fcc and hcp equations of state are well reproduced. On the other hand, the bcc, sc and diamond structures were found to collapse under an NPT simulation, even at temperatures of a few ten $K$, and can be regarded as unstable. Because of the functional form chosen for the potential, we were not able to fit the hcp-fcc energy difference nor the elastic constants (see table 2), but as the intended application is geared towards surface phenomena, this was not considered a major drawback. However, the bulk modulus obtained with the optimized potential reproduced roughly the experimental value. Of the DFT methods, LDA gives a clearly better description of the equation of state of fcc gold, the bulk modulus being $6 \%$ higher than the experimental value. PBE on the other hand underestimates the value by $25 \%$ (see table 2 ). Note, however, that the effect of zero point vibrations was ignored.

All of the reviewed gold potentials were fitted to the elastic constants, reproducing roughly either the experimental room temperature or the $0 \mathrm{~K}$ bulk modulus (see table 2). We shall not review the bulk properties of other potentials in detail, however, as they are usually readily available in the original publications, and differences between them are small.

Simulating bulk fcc gold with the present potential gave a lattice constant of $4.07 \AA$ at $300 \mathrm{~K}$ with a thermal expansion coefficient of $\sim 9 \cdot 10^{-6} \mathrm{~K}^{-1}$, the experimental value being $14 \cdot 10^{-6} \mathrm{~K}^{-1}$ [37]. The thermal expansion seems to be somewhat weaker compared to the FBD, GRS, and Glue potentials for which it was evaluated in ref. [36].

The melting point of gold with the optimized potential was determined using a standard procedure where the evolution of a half crystalline, half molten system was followed at different temperatures to see whether the solid or molten phase would start to grow. The crystalline side of the solid-melt interface was a (100) surface. A melting point of $(2125 \pm 25) \mathrm{K}$ was obtained. The fact that the value is high can be understood by the too high values of the vacancy formation energy $\left(E_{\mathrm{vac}}\right)$ and $\mathrm{c}_{44}$ (see table 2$)$, since these properties have been shown to have an indirect correlation with the melting point $[38,39,40,41,42]$.

\subsection{Surface properties}

Surface energies are hard to obtain accurately with many interatomic potentials. Embedded-atom-method-type potentials for example often underestimate them since they were generated to describe bulk properties. This is also the case for the present potential, although the underestimation is not too severe. Energies for the (100) and (111) surfaces are shown in table 3 and compared to other po- 


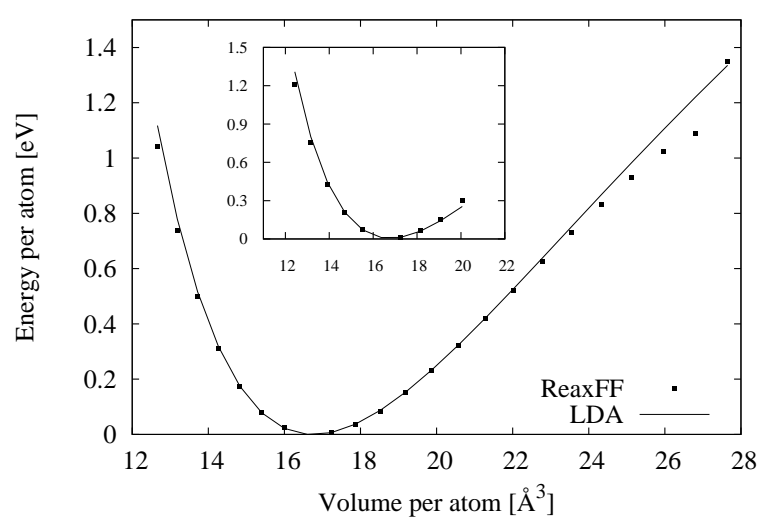

Figure 1. Equations of state for fcc and hcp (inset) gold. The energies are with respect to the fcc equilibrium.
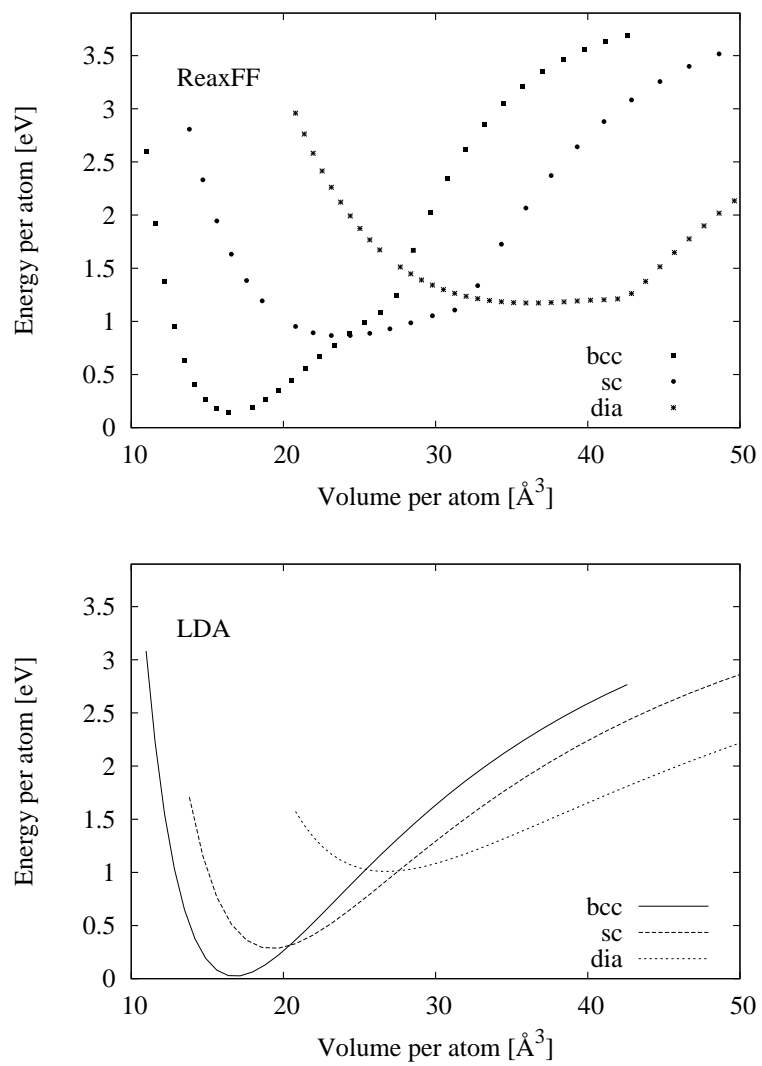

Figure 2. Equations of state for bcc, sc, and diamond structured gold. The energies are with respect to the fcc equilibrium. tentials. The DFT results in the table were obtained with VASP using a four-layer slab model with $3 \times 3$ unit cells for the (100) and $4 \times 2$ for the (111) surface. (The (111) unit cells refer to orthogonal ones with 2 atoms per layer.) These slabs resulted in surface defect coverages of about $6 \%$. A $k$-point sampling of $8 \times 8 \times 1$ and an energy cutoff of $520 \mathrm{eV}$ was used, giving convergence to about \pm 1 for the last given digit in table 3 .

The $\mathrm{Au}(100)$ surface reconstructs in a hexagonal-like pattern for which the surface energy is $\sim 0.04 \mathrm{~J} / \mathrm{m}^{2}$ lower than that for the unreconsructed surface [46]. As a model of the reconstruction, we used the minimal commensurate $\mathrm{p}(1 \times 5)$ unit cell provided by ref. [47]. The reconstruction is unfortunately unstable with the present potential, the first layer atoms occupying adatom positions after relaxation.

Out of the other potentials considered here, the glue model gives correct behaviour, although the reconstruction is somewhat overstabilized, the energy difference to the unreconstructed surface being $0.4 \mathrm{~J} / \mathrm{m}^{2}$. None of the other potentials reproduced the reconstruction, relaxing into configurations similar to that given by the present one $[48,36,49]$. However, while the glue model was correct in this respect, its transferability is limited, as it gives unphysical behaviour for the dimer and the (111) adatom (see below).

Besides surfaces, important low-coordinated cases are the adatom and surface vacancy formation energies. Table 3 shows the cases of (100) and (111) surfaces. The surface vacancy energies are defined as $E_{\mathrm{vac}}=E_{\mathrm{ads}}+E_{\mathrm{coh}}$ 
and the adatom energies as $E_{\text {adat }}=E_{\text {des }}-E_{\text {coh }}$, where $E_{\text {ads }}$ is the adsorption energy and $E_{\text {des }}$ the desorption energy, that is, the energy change required to bring an atom to the surface from vacuum, and to remove one from the surface. Note that for the DFT methods, the energy given by VASP for an atom in vacuum $(-0.29 \mathrm{eV}$ for PBE and $-0.12 \mathrm{eV}$ for LDA) was substracted from all physical energies. With the glue potential, the (100) adatom was not stable, being pulled into the surface to an interstitial-like position. The same was true for the (111) fcc adatom. It seems that these effects are related to the too high stabilization of the reconstructed (100) surface.

\subsection{Dimer}

The dimer provides a stringent test for potential properties at low coordination. The energy vs. distance curve is shown in fig. 3 and the equilibrium values in table 4 . Values calculated with other potentials and DFT are shown for comparison.

The present potential gives a good description of the equilibrium and repulsive parts of the dimer curve, but the dissociation happens in a somewhat short interval. Of the other potentials, FBD gives a too tightly bound and short dimer, while Voter and CEM (not shown) give very good reproduction of DFT data. Of these, at least CEM was fit to the dimer. Surprisingly, the glue potential gives a completely unphysical dimer. This seems to be the reason it was found to underbind monatomic gold wires in ref. [51]. Also the GRS dimer is severely underbound, with a spurious minimum at around $2 \AA$.

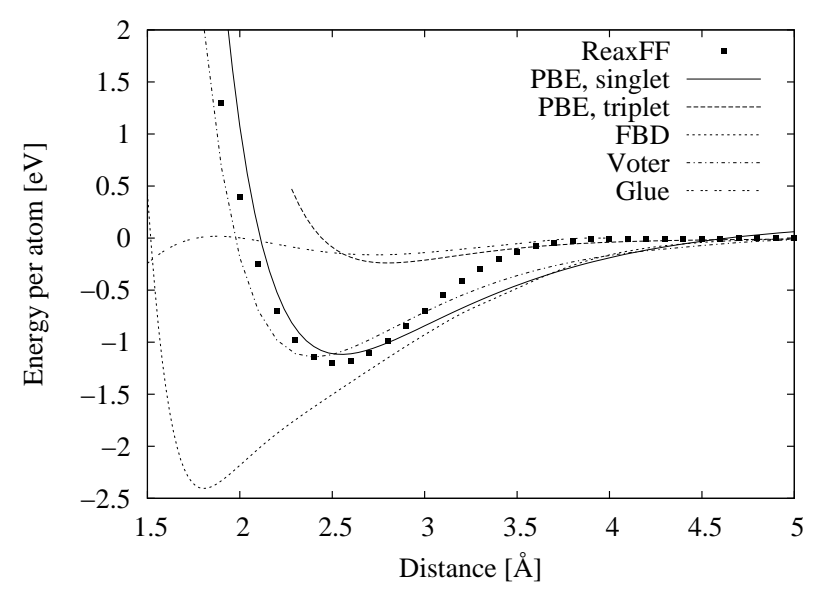

Figure 3. Dimer dissociation curves as calculated by the present potential, other potentials, and PBE-DFT.

\section{Conclusions}

We have developed a potential to describe gold in the ReaxFF framework, with emphasis on low-coordinated systems. The potential parameters are available as supporting information for this article. The surface energetics of the potential compare very well with other potentials and density functional calculations, and the gold dimer is well reproduced. The present work is also an important step towards extending the range of ReaxFF interaction towards complete simulation of gold-thiol chemistry, as well as other applications where gold surfaces and particles play a crucial role.

We would like to thank Dr. H. Häkkinen for useful discussions. This work was performed within the Finnish Centre of Excellence in Computational Molecular Science (CMS), financed by the Academy of Finland and the University of Helsinki. We also gratefully acknowledge the grants of computer time from CSC, the Finnish IT centre for science. T. Jacob gratefully acknowledges support 
by the "Deutsche Forschungsgemeinschaft" (DFG) within the Emmy-Noether-Program, the "Fonds der Chemischen Industrie" (FCI), and the Alexander von Humboldt foundation $(\mathrm{AvH})$.

\section{A Potential functions and parameters}

In this appendix, we detail the interactions that were used in fitting the present potential for gold (see eq. (1)), as well as the optimized parameters. The bond energy is

$$
E_{\mathrm{bond}}=-D_{e}^{\sigma} B O_{i j}^{\sigma} \exp \left[p_{b e 1}\left(1-\left(B O_{i j}^{\sigma}\right)^{p_{b e 2}}\right)\right]
$$

where the bond-order of the bond between atoms $i$ and $j$ is given by

$$
B O_{i j}^{\sigma}=\exp \left[p_{b o 1}\left(\frac{r_{i j}}{r_{0}^{\sigma}}\right)^{p_{b o 2}}\right] .
$$

The van der Waals energy is described by a distancecorrected Morse potential and is given by

$$
\begin{aligned}
E_{\mathrm{vdW}}= & T\left(r_{i j}\right) D_{v d W}\left\{\exp \left[\alpha_{i j}\left(1-\frac{f_{13}\left(r_{i j}\right)}{r_{v d W}}\right)\right]\right. \\
& \left.-2 \exp \left[\frac{1}{2} \alpha_{i j}\left(1-\frac{f_{13}\left(r_{i j}\right)}{r_{v d W}}\right)\right]\right\},
\end{aligned}
$$

where

$$
f_{13}\left(r_{i j}\right)=\left[r_{i j}^{p_{v d W 1}}+\gamma_{w}^{-p_{v d W 1}}\right]^{\frac{1}{p_{v d W 1}}}
$$

and the Taper correction, taking the term smoothly to zero at a cutoff distance, $R_{\text {cut }}$, of $10 \AA$, is given by

$$
T(r)=\frac{20}{R_{\mathrm{cut}}^{7}} r^{7}-\frac{70}{R_{\mathrm{cut}}^{6}} r^{6}+\frac{84}{R_{\mathrm{cut}}^{5}} r^{5}-\frac{35}{R_{\mathrm{cut}}^{5}} r^{5}+1 .
$$

The overcoordination correction for atom $i$ is calculated as

$$
E_{\text {over }}=\frac{\sum_{j=1}^{n \text { bond }} p_{\text {ovun } 1} D_{e}^{\sigma} B O_{i j}}{\Delta+\operatorname{Val}} \Delta\left[1+\exp \left(p_{\text {ovun } 2} \Delta\right)\right]^{-1},
$$

where

$$
\Delta=-\mathrm{Val}+\sum_{j=1}^{n \text { nbonds }} B O_{i j},
$$

and the sums are over the bonds from atom $i$ to its neighbours.

The potential parameters are given in table 5 .

\section{References}

1. J.C. Love, L.A. Estroff, J.K. Kriebel, R.G. Nuzzo, G.M. Whitesides, Chem. Rev. 105, 1103 (2005)

2. K.M. Beardmore, J.D. Kress, N. Grønbech-Jensen, A.R. Bishop, Chem. Phys. Lett. 286, 40 (1998)

3. B. Jeon, J.D. Kress, N. Grønbech-Jensen, Phys. Rev. B 76, $155120(2007)$

4. S. Jiang, Mol. Phys. 100(14), 2261 (2002)

5. L. Zhang, W.A. Goddard III, S. Jiang, J. Chem. Phys. $\mathbf{1 1 7}(15), 7342(2002)$

6. A.C.T. van Duin, S. Dasgupta, F. Lorant, W.A. Goddard III, J. Phys. Chem. A 105(41), 9396 (2001)

7. K. Chenoweth, A.C.T. van Duin, W.A. Goddard, III, J. Phys. Chem. A 112, 1040 (2008)

8. Q. Zhang, T. Çağin, A.C.T. van Duin, W.A. Goddard III, Y. Qi, J. Louis G. Hector, Phys. Rev. B 69, 045423 (2004)

9. J. Ludwig, D.G. Vlachos, A.C.T. van Duin, W.A. Goddard, III, J. Phys. Chem. B 110, 4274 (2006)

10. S.S. Han, A.C.T. van Duin, W.A. Goddard III, H.M. Lee, J. Phys. Chem. A 109(20), 4575 (2005)

11. W.A. Goddard III, A. van Duin, K. Chenoweth, M.J. Cheng, S. Pudar, J. Oxgaard, B. Merinov, Y.H. Jang, P. Persson, Topics in Catalysis 38(1-3), 93 (2006)

12. K.D. Nielson, A.C.T. van Duin, J. Oxgaard, W.Q. Deng, W.A. Goddard III, J. Phys. Chem. A 109, 493 (2005) 
13. G. Kresse, J. Hafner, Phys. Rev. B 47, 558 (1993)

14. G. Kresse, J. Hafner, Phys. Rev. B 49, 14251 (1994)

15. G. Kresse, J. Furthmüller, Phys. Rev. B 54, 11169 (1996)

16. G. Kresse, J. Furthmüller, Comput. Mat. Sci. 6(1), 15 (1996)

17. P.E. Blöchl, Phys. Rev. B 50, 17953 (1994)

18. G. Kresse, D. Joubert, Phys. Rev. B 59, 1758 (1999)

19. J.P. Perdew, K. Burke, M. Ernzerhof, Phys. Rev. Lett. $77(18), 3865(1996)$

20. H. Häkkinen, U. Landman, Phys. Rev. B 62(4), R2287 (2000)

21. H. Häkkinen, B. Yoon, U. Landman, X. Li, H.J. Zhai, L.S. Wang, J. Phys. Chem. A 107, 6168 (2003)

22. X. Gu, M. Ji, S.H. Wei, X.G. Gong, Phys. Rev. B 70, $205401(2004)$

23. M.J. Frisch, G.W. Trucks, H.B. Schlegel, G.E. Scuseria, M.A. Robb, J.R. Cheeseman, J. J. A. Montgomery, T. Vreven, K.N. Kudin, J.C. Burant et al., Gaussian O3, Revision C.02, Gaussian, Inc., Wallingford CT, 2004

24. H. Basch, M.A. Ratner, J. Chem. Phys. 119(22), 11926 (2003)

25. J.F. Ziegler, J.P. Biersack, U. Littmark, The Stopping and Range of Ions in Matter (Pergamon, New York, 1985)

26. S.M. Foiles, M.I. Baskes, M.S. Daw, Phys. Rev. B 33(12), 7983 (1986)

27. F. Ercolessi, M. Parrinello, E. Tosatti, Phil. Mag. A 58(1), $213(1988)$

28. M.S. Stave, D.E. Sanders, T.J. Raeker, A.E. DePristo, J. Chem. Phys. 93(6), 4413 (1990)

29. S.B. Sinnott, M.S. Stave, T.J. Raeker, A.E. DePristo, Phys. Rev. B 44(16), 8927 (1991)
30. T.J. Raeker, A.E. DePristo, Int. Rev. Phys. Chem. 10(1), $1(1991)$

31. A.F. Voter, Los Alamos Unclassified Technical Report \#LA-UR-93-3901 (1993)

32. F. Cleri, V. Rosato, Phys. Rev. B 48(1), 22 (1993)

33. J. Cai, Y.Y. Ye, Phys. Rev. B 54(12), 8398 (1996)

34. G.C. Kallinteris, N.I. Papanicolaou, G.A. Evangelakis, D.A. Papaconstantopoulos, Phys. Rev. B 55(4), 2150 (1997)

35. H. Chamati, N.I. Papanicolaou, J. Phys.: Condens. Matt. 16, 8399 (2004)

36. G. Grochola, S.P. Russo, I.K. Snook, J. Chem. Phys. 123, 204719 (2005)

37. D.R. Lide, ed., CRC Handbook of Chemistry and Physics, Internet Version, 88th edn. (Taylor and Francis, 2007)

38. L. Brillouin, Phys. Rev. 54, 916 (1938)

39. G.E. Jelinek, Phys. Rev. B 3(8), 2724 (1971)

40. A.V. Granato, Phys. Rev. Lett. 68(7), 974 (1992)

41. M. Forsblom, G. Grimvall, Phys. Rev. B 72, 054107 (2005)

42. C.C. Matthai, N.H. March, Phys. Chem. Liq. 44(3), 329 $(2006)$

43. E.A. Brandes, G.B. Brook, eds., Smithells Metals Reference Book, 7th edn. (Butterworth-Heinemann, Oxford, UK, 1992)

44. O.L. Anderson, D.G. Isaak, S. Yamamoto, J. Appl. Phys. 65(4), $1534(1989)$

45. H.E. Schaefer, Phys. Stat. Sol. A 102, 47 (1987)

46. T. Jacob, Electrochim. Acta 52, 2229 (2007)

47. B.W. Dodson, Phys. Rev. B 35(2), 880 (1987)

48. F. Ercolessi, E. Tosatti, M. Parrinello, Phys. Rev. Lett. $\mathbf{5 7}(6), 719$ (1986)

49. M.I. Haftel, Phys. Rev. B 48(4) (1993) 
50. W.R. Tyson, W.A. Miller, Surf. Sci. 62, 267 (1977)

51. J.A. Torres, E. Tosatti, A.D. Corso, F. Ercolessi, J.J. Kohanoff, F.D.D. Tolla, J.M. Soler, Surf. Sci. Lett. 426, L441 (1999)

\begin{tabular}{|c|c|c|c|}
\hline property & ReaxFF & lit. & ref. \\
\hline \multirow[t]{3}{*}{$E_{\mathrm{coh}} / \mathrm{eV}$} & 3.91 & 3.93 & {$[43]$} \\
\hline & & 4.28 & LDA \\
\hline & & 2.98 & PBE \\
\hline$E_{\mathrm{hcp}}-E_{\mathrm{coh}} / \mathrm{eV}$ & 0.0002 & 0.0054 & LDA \\
\hline$E_{\mathrm{bcc}}-E_{\mathrm{coh}} / \mathrm{eV}$ & 0.14 & 0.026 & LDA \\
\hline$E_{\mathrm{sc}}-E_{\mathrm{coh}} / \mathrm{eV}$ & 0.86 & 0.28 & LDA \\
\hline$E_{\mathrm{dia}}-E_{\mathrm{coh}} / \mathrm{eV}$ & 1.17 & 1.01 & LDA \\
\hline \multirow[t]{3}{*}{$a_{\mathrm{fcc}} / \AA$} & 4.06 & 4.078 & {$[37](\mathrm{RT})$} \\
\hline & & 4.06 & LDA \\
\hline & & 4.17 & $\mathrm{PBE}$ \\
\hline$a_{\text {bcc }} / \AA$ & 3.21 & 3.23 & LDA \\
\hline$a_{\mathrm{sc}} / \AA$ & 2.87 & 2.68 & LDA \\
\hline$a_{\text {dia }} / \AA$ & 6.62 & 5.98 & LDA \\
\hline$a_{\mathrm{hcp}} / \AA$ & 2.87 & 2.85 & LDA \\
\hline$c_{\mathrm{hcp}} / \AA$ & 4.69 & 4.77 & LDA \\
\hline $\mathrm{c}_{11} / 10^{12} \mathrm{erg} / \mathrm{cm}^{3}$ & 2.49 & 1.92 & {$[37](\mathrm{RT})$} \\
\hline $\mathrm{c}_{12} / 10^{12} \mathrm{erg} / \mathrm{cm}^{3}$ & 1.42 & 1.63 & {$[37](\mathrm{RT})$} \\
\hline $\mathrm{c}_{44} / 10^{12} \mathrm{erg} / \mathrm{cm}^{3}$ & 1.47 & 0.42 & [37] $(\mathrm{RT})$ \\
\hline \multirow[t]{4}{*}{$\mathrm{B} / \mathrm{GPa}$} & 177 & 180 & {$[44]$} \\
\hline & & 173 & {$[37](\mathrm{RT})$} \\
\hline & & 190 & LDA \\
\hline & & 135 & PBE \\
\hline$E_{\mathrm{vac}} / \mathrm{eV}$ & 1.79 & 0.89 & {$[45,34]$} \\
\hline
\end{tabular}

Table 2. Bulk properties of the potential, evaluated at $0 \mathrm{~K}$.

The reference values are at $0 \mathrm{~K}$ unless room temperature (RT) is specified. 


\begin{tabular}{|c|c|c|c|c|c|c|}
\hline potential & $\mathrm{r} / \AA$ & $\mathrm{E} / \mathrm{eV} /$ atom & ref. / note & \multicolumn{3}{|c|}{ General parameters } \\
\hline expt. & 2.47 & 1.17 & {$[37]$} & parameter & \multicolumn{2}{|c|}{ value } \\
\hline ReaxFF & 2.52 & 1.20 & & $p_{v d W 1}$ & \multicolumn{2}{|c|}{1.5591} \\
\hline PBE & 2.55 & 1.12 & & \multirow{2}{*}{\multicolumn{3}{|c|}{ Atom parameters }} \\
\hline $\mathrm{LDA}$ & 249 & 1.44 & & & & \\
\hline & & & & parameter & \multicolumn{2}{|c|}{ value } \\
\hline $\mathrm{CP}$ & 2.58 & 1.47 & & $r_{0}^{\sigma} / \AA$ & \multicolumn{2}{|c|}{2.0271} \\
\hline C-R & 2.31 & 2.42 & & $p_{\text {ovun } 2}$ & -24.7561 & \\
\hline $\mathrm{CY}$ & 2.39 & 2.80 & & Val & \multicolumn{2}{|c|}{1} \\
\hline FBD & 1.81 & 2.41 & & \multicolumn{3}{|c|}{ van der Waals parameters } \\
\hline Glue & 2.74 & 0.16 & 2nd min. at $0.6 \mathrm{eV}, 1.2 \AA$ & \multicolumn{2}{|c|}{ parameter } & value \\
\hline GRS & 2.72 & 0.31 & 2nd min. at $0.16 \mathrm{eV}, 2.0 \AA$ & \multicolumn{2}{|c|}{$r_{v d W} / \AA$} & 4.4156 \\
\hline KAL & 2.27 & 2.78 & & \multicolumn{2}{|c|}{$D_{v d W} / \mathrm{kcal} / \mathrm{mol}$} & 0.3446 \\
\hline Voter & 2.40 & 1.14 & & \multicolumn{2}{|c|}{$\alpha_{v d W}$} & 11.9754 \\
\hline & & & & \multicolumn{2}{|c|}{$\gamma_{v d W} / \AA^{-1}$} & 2.0434 \\
\hline
\end{tabular}

Table 4. Comparison of dimer properties of the different po-

Bond parameters tentials and density functional methods.

\begin{tabular}{c|c}
\multicolumn{2}{c|}{ Bond parameters } \\
parameter & value \\
\hline$D_{e}^{\sigma} / \mathrm{kcal} / \mathrm{mol}$ & 146.6542 \\
$p_{\text {bo } 1}$ & -0.1591 \\
$p_{\text {bo } 2}$ & 5.3892 \\
$p_{\text {be } 1}$ & -0.0295 \\
$p_{\text {be } 2}$ & 0.2793 \\
$p_{\text {ovun } 1}$ & 0.3319
\end{tabular}

Table 5. Force field parameters. The general parameters were not subjected to fitting, as they are common for a class of potentials. 


\begin{tabular}{c|ccccccccccccccc} 
property & ReaxFF & GRS & Glue & FBD & Voter & C-R & CEM & KAL & CP & CY & LDA & PBE & expt. \\
\hline$\gamma_{(100)}$ & 1.21 & 1.30 & 2.06 & 0.91 & 0.89 & 0.58 & 1.55 & 0.44 & 1.05 & 0.68 & 1.35 & 0.87 \\
$\gamma_{(111)}$ & 1.07 & 1.20 & 1.55 & 0.79 & 0.77 & 0.49 & 1.44 & 0.37 & 0.93 & 0.62 & 1.13 & 0.71 \\
$\gamma$ & & & & & & & & & & & & & \\
\hline & & & & & & & & & & & & & \\
$E_{\text {adat(100) }}$ & 0.59 & 0.99 & -6.3 & 0.43 & 0.54 & 0.30 & 0.86 & 0.16 & 0.68 & 0.31 & 0.30 & 0.14 \\
$E_{\text {vac(100) }}$ & 0.59 & 0.95 & -7.8 & 0.44 & 0.46 & 0.27 & 0.80 & 0.17 & 0.59 & 0.32 & 0.40 & 0.31 \\
$E_{\text {adat(111),fcc }}$ & 0.843 & 1.51 & -0.05 & 0.899 & 1.077 & 0.601 & 1.217 & 0.425 & 1.020 & 0.511 & 0.95 & 0.61 \\
$E_{\text {adat(111),hcp }}$ & 0.843 & 1.50 & 0.25 & 0.897 & 1.079 & 0.600 & 1.217 & 0.425 & 1.021 & 0.512 & 1.00 & 0.64 \\
$E_{\text {vac(111) }}$ & 0.87 & 1.10 & 1.28 & 0.70 & 0.70 & 0.45 & 0.98 & 0.31 & 0.79 & 0.46 & 0.88 & 0.61
\end{tabular}

Table 3. Comparison of clean surface, adatom, and surface vacancy energies of the different potentials and density functional methods. All surface energies are in $\mathrm{J} / \mathrm{m}^{2}$ and all defect energies in $\mathrm{eV}$. 